\title{
Lack of the Association of the PTPN22 C1858T Gene Polymorphism With Susceptibility to Familial Mediterranean Fever
}

\author{
Orhan KÜÇÜKŞAHIN,,${ }^{1}$ Zeynep ŞEKER, ${ }^{2}$ Ali ŞAHIN,,${ }^{4}$ Gülay KINIKLI, ${ }^{1}$ Timur TUNCALI, ${ }^{3}$ Murat TURGAY, ${ }^{1}$ \\ Alexis K OKOH, ${ }^{2}$ Emre KÜLAHÇIOĞLU, ${ }^{2}$ Şükran ERTEN, ${ }^{5}$ Aşkın ATEŞ ${ }^{1}$ \\ ${ }^{1}$ Department of Internal Medicine, Division of Rheumatology, Medical Faculty of Ankara University, Ankara, Turkey \\ ${ }^{2}$ Department of Internal Medicine, Medical Faculty of Ankara University, Ankara, Turkey \\ ${ }^{3}$ Department of Medical Genetics, Medical Faculty of Ankara University, Ankara, Turkey \\ ${ }^{4}$ Department of Rheumatology, Medical Faculty of Cumhuriyet University, Sivas, Turkey \\ ${ }^{5}$ Department of Rheumatology, Yıldırım Beyazıt University Atatürk Training and Research Hospital, Ankara, Turkey
}

\begin{abstract}
Objectives: This study aims to investigate whether the protein tyrosine phosphatase non-receptor type 22 (PTPN22) C1858T gene polymorphism plays a role in the pathogenesis of familial Mediterranean fever (FMF) through T-lymphocyte activation.

Patients and methods: We conducted a case-control study with 180 FMF patients ( 68 males, 112 females; mean age $38.2 \pm 1.6$ years; range 16 to 81 years) and 184 healthy controls ( 86 males, 98 females; mean age $32.9 \pm 9.2$ years; range 18 to 58 years). The PTPN22 C1858T polymorphism (rs2476601) was genotyped by polymerase chain reaction restriction fragment length polymorphism. In patients with FMF, clinical features, disease severity score, the frequencies of amyloidosis, positive family history, and Mediterranean fever gene mutations were determined.

Results: The frequencies of heterozygous genotype (CT) were $4.5 \%$ in FMF patients and $2.8 \%$ in healthy controls, respectively. The frequencies of polymorphic homozygous genotypes (TT) were $0.5 \%$ in both FMF patients and healthy controls. There were no statistically significant differences in the frequencies of CT and TT genotypes between FMF patients and healthy controls (odds ratio: $1.65,95 \%$ confidence interval: $0.53-5.14, p>0.05$ for CT genotype). The frequencies of clinical features, sex, amyloidosis, positive family history, Mediterranean fever gene mutations, and disease severity score were not significantly different between the patients.

Conclusion: The distribution of PTPN22 C1858T polymorphism did not reveal any association with FMF in a Turkish population.

Keywords: Familial Mediterranean fever; protein tyrosine phosphatase non-receptor type 22; single nucleotide polymorphism.
\end{abstract}

Familial Mediterranean fever (FMF) is an autosomal recessively inherited auto-inflammatory disease seen frequently in communities around the Mediterranean Sea and characterized by periodic attacks of fever accompanied by inflammation localized to serosal surfaces, synovium or skin. ${ }^{1,2}$ Pyrin or marenostrin are products of the Mediterranean fever (MEFV) gene, a regulatory protein that is mainly expressed in neutrophils. It has been revealed that pyrin plays a regulatory role in the inflammatory pathway by leading inactivation of activated neutrophils and apoptosis.
Pyrin dysfunction caused by upregulation of the MEFV gene is thought to be important in the etiopathogenesis of FMF. ${ }^{3}$ Several studies have also demonstrated a T-helper 1 (Th1) polarization in the inflammatory response of FMF. These studies suggest that the $\mathrm{T}$ cell system is activated abnormally in FMF patients. ${ }^{4-6}$

The C1858T substitution resulting in an arginine to tryptophan change in codon 620 of the protein product has been shown to affect the protein-protein interaction with tyrosine kinase in 
T-cell activation, reducing the interaction between the protein and tyrosine kinase, resulting in an increased risk of inappropriate T-cell activation. ${ }^{7}$ A functional polymorphism located in the coding region of protein tyrosine phosphatase non-receptor type 22 (PTPN22), the gene that encodes the intracellular PTPN 22 has been suggested to increase the risk for developing several autoimmune diseases, such as type I diabetes, systemic lupus erythematosus, Graves' disease, Hashimato thyroiditis, and rheumatoid arthritis (RA). ${ }^{8-11}$

It is suggested that the PTPN22 gene polymorphism causes a tendency for $\mathrm{T}$ lymphocyte dysfunction in many autoimmune diseases. Although it is suggested that monocytes and macrophages are the main cells that are responsible for the disease, activated T lymphocytes may also contribute to the pathogenesis of FMF. In this study, we aimed to investigate whether PTPN22 $\mathrm{C} 1858 \mathrm{~T}$ gene polymorphism plays a role in the pathogenesis of FMF through $\mathrm{T}$ lymphocyte activation.

\section{PATIENTS AND METHODS}

A case-control study was conducted at Ankara University Medical Faculty, Department of Rheumatology, Turkey, between March 01, 2008 and March 01, 2009. One-hundred eighty patients with FMF (68 males, 112 females; mean age $38.2 \pm 1.6$ years; range 16 to 81 years) and 184 blood bank donors that served as healthy controls

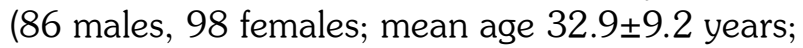
range 18 to 58 years) from the same hospital were included in the study. The mean disease duration was $113 \pm 87$ months in patients with FMF. All patients and controls were enrolled with local ethics committee approval and provided their informed consent. The study was conducted in accordance with the principles of the Declaration of Helsinki. The diagnosis of patients with FMF was made using criteria suggested by Livneh et al. ${ }^{12}$ The patients with autoinflammatory diseases, chronic inflammatory and autoimmune diseases except FMF were excluded. Detailed clinical features and demographic data were recorded for each patient. The presence of amyloidosis, positive family history of FMF, and MEFV gene mutations were also detected. Disease severity was evaluated by using severity scoring system for FMF by Pras et al. ${ }^{13}$ The frequencies of clinical features, amyloidosis, positive family history of FMF, MEFV gene mutations, and disease severity are shown in the Table 1.

Genomic deoxyribonucleic acid samples were extracted from peripheral blood by a commercial kit Nucleospin Blood QuickPure, (Macherey-Nagel, Düren, Germany) following manufacturer's protocol. The fragment that contains the 1858C >T polymorphism (rs2476601) was amplified in $1 \mathrm{X}$ Tris-hydrochloride, boric acid, Ethylenediaminetetraacetic acid reaction buffer (AppliChem $\mathrm{GmbH}$, Darmstat, Germany) containing $1.5 \mathrm{mM} \mathrm{MgCl} 2,200 \mu \mathrm{M}$ deoxynucleotide mix (MBI-Fermentas, Burlington, Canada), $1 \mathrm{U}$ of Taq deoxyribonucleic acid polymerase (MBI, Berlin, Germany), 10 pmol of each of the primers: forward primer, 5-AGCAAA AACCTCCTGGGTTTGTACC-3'; reverse primer, 5'-AGGAAGCTGGTGAGTACAGTTCAGT-3' and $1 \mu \mathrm{L}$ of sample deoxyribonucleic acid in a total volume of $50 \mu \mathrm{L}$. The amplification reaction was set to 30 cycles of one minute denaturation at $95{ }^{\circ} \mathrm{C}$; one minute annealing at $58{ }^{\circ} \mathrm{C}$; one minute elongation at $72{ }^{\circ} \mathrm{C}$ starting with five minutes of initial denaturation at $95^{\circ} \mathrm{C}$.

Table 1. Clinical features of patients with familial Mediterranean fever

\begin{tabular}{lccc}
\hline & $\mathrm{n}$ & $\%$ & Mean $\pm \mathrm{SD}$ \\
\hline Age (years) & & & $38.2 \pm 1.6$ \\
Sex & 112 & 62.2 & \\
$\quad$ Female & 68 & 37.8 & \\
$\quad$ Male & 169 & 93.8 & \\
Fever & 175 & 97.2 & \\
Abdominal pain & 89 & 49.4 & \\
Arthritis & 57 & 32.2 & \\
Pleuritis & 7 & 3.8 \\
Pericarditis & 22 & 12.2 & \\
Erysipelas & 28 & 15.5 & \\
Amyloidosis & 88 & 48.8 & \\
Family history of FMF & 175 & 97.2 & \\
Colchicine usage & & & \\
Disease severity & 57 & 32.2 & \\
$\quad$ Mild & 79 & 44.6 & \\
Moderate & 41 & 23.2 & \\
Severe & & & \\
MEFV gene mutation carriage & 17 & 16.5 & \\
$\quad$ M694V homozygote & 4 & 3.9 & \\
M680I homozygote & 3 & 2.9 & \\
V726A homozygote & 56 & 54.4 & \\
Compound heterozygote & 23 & 22.3 & \\
$\quad$ Other mutations & & & \\
\hline SD: Standard deviation; FMF: & Familial & Mediterranean fever; \\
MEFV: Mediterranean fever gene. & & & \\
\hline
\end{tabular}


The amplified products then were digested in separate reactions with Rsal (MBI-Fermentas, Burlington, Canada) and $\mathrm{XcmI}$ restriction endonucleases overnight at $37{ }^{\circ} \mathrm{C}$. Digested products were separated in $2.5 \%$ agarose gel electrophoresis. Fragments were visualized and analyzed under ultraviolet illumination (Kodak, Wilmington, North Carolina, USA). Wild type alleles (1858C) yielded 228 bp fragments with RsaI digestion and 304 bp fragments with $\mathrm{XcmI}$ digestion. Whereas polymorphic alleles (1858T) had 272 bp fragments.

The required sample size for $90 \%$ power, $\alpha=0.05$ type I error, $\beta=0.10$ type II error and $\mathrm{d}=0.17$ effect size was calculated as minute for 356 total subjects.

\section{Statistical analysis}

SPSS version 11.0 package program for Windows (SPSS Inc., Chicago, IL, USA) was used for the data analysis. Patients and controls were summarized for their genotypes, clinical features, and demographic properties as mean \pm standard deviation for quantitative variables. Descriptive statistics were applied with mean \pm standard deviation and median values for variables that did not fit to normal distribution. Mann-Whitney $U$ and Chi-square tests were used for the analysis of nonnormally and normally distributed independent variables, respectively. A $p<0.05$ was considered statistically significant.

\section{RESULTS}

We examined PTPN22 C1858T polymorphism in 177 FMF patients and 180 healthy controls. The frequencies of PTPN22 C1858 T polymorphism in patients with FMF and healthy controls are shown in Table 2. Genotype frequencies for the PTPN22 C1858T polymorphism were in
Hardy-Weinberg equilibrium in both the patient and the healthy control groups. The frequencies of heterozygous genotype (CT) were $4.5 \%$ in FMF patients and $2.8 \%$ in healthy controls, respectively. The frequencies of polymorphic homozygous genotype (TT) were $0.5 \%$ in both FMF patients and the healthy controls. There were no statistically significant differences between FMF patients and healthy controls according to the frequencies of $\mathrm{CT}$ and $\mathrm{TT}$ genotypes, respectively (odds ratio: 1.65, 95\% confidence interval: $0.53-5.14, \mathrm{p}>0.05$ for $\mathrm{CT}$ ) (Table 2). The frequencies of clinical features, sex, amyloidosis, positive family history, MEFV gene mutations and disease severity were not significantly different between the patients with or without PTPN22 $\mathrm{C} / \mathrm{T}$ or $\mathrm{T} / \mathrm{T}$ genotypes.

\section{DISCUSSION}

In previous studies, PTPN22 gene polymorphism has been shown to be related with many T-cell mediated autoimmune diseases such as type I diabetes mellitus, systemic lupus erythematosus, Graves' disease, Hashimato thyroiditis, and RA. ${ }^{8-11}$ However, there are also some inflammatory diseases such as inflammatory bowel disease, ankylosing spondylitis, multiple sclerosis and Behçet's disease with no association of PTPN22 C1858T polymorphism. ${ }^{14-16}$ FMF is characterized by periodic attacks of fever accompanied by inflammation localized to serosal surfaces, synovium, or skin. It has been suggested that there seems to be an ongoing subclinical inflammation in FMF patients during an attack-free period. ${ }^{17}$

T-helper 1 type polarization has been implicated in the pathogenesis of FMF. ${ }^{4}$ Musabak et al. ${ }^{5}$ has previously demonstrated that $\mathrm{T}$ cells are abnormally activated in patients with FMF, either when symptomatic or between the

\begin{tabular}{l} 
Table 2. Genotype frequencies of protein tyrosine phosphatase non-receptor type 22 \\
C1858T polymorphism in patients with familial Mediterranean fever and healthy controls \\
\hline Genotype \\
\cline { 2 - 7 }
\end{tabular}


attacks, and Th1 differentiation is the hallmark of inflammations in those patients. According to the study by Simsek et al., ${ }^{6}$ increased levels of interleukin-12 and interleukin-18 in patients with inactive disease imply that they seem to assist Th1 activation and subclinical inflammation persisting during the attack-free period of the disease. In this study, we examined the possible role of the PTPN22 gene polymorphism responsible for inappropriate $\mathrm{T}$ cell activation in patients with FMF. We did not find significant differences in the frequencies of CT and TT genotypes between FMF patients and healthy controls.

The genetic studies performed in various populations revealed that there are geographic differences in the frequency of PTPN22 C1858T genotypes. The frequency of PTPN22 1858T allele was highest in Finland (15.5\%), Sweden (12\%), China (8\%), Spain (6\%), and Italy (2\%) whereas PTPN22 $1858 \mathrm{~T}$ allele was very rare in Asian (9\%), African (3\%), and American (8\%) communities. ${ }^{18}$ Furthermore, PTPN22 1858 CT and TT genotype frequencies in both several autoimmune diseases and healthy controls in European and North American ancestry are higher than those in Asian populations. ${ }^{8-11,19-21}$ We previously reported that the frequency of PTPN22 C1858T CT polymorphism in a Turkish population $(8.4 \%$ in $\mathrm{RA}$ patients and $5.4 \%$ in healthy controls) was also lower than those in Caucasian populations. Moreover, the TT genotype was absent in both RA patients and the healthy controls. ${ }^{22}$ Similarly, previous studies conducted in Turkish populations showed that the frequency of the PTPN22 polymorphism is relatively low; ${ }^{16,22}$ in this study, the frequencies of PTPN22 1858 CT and TT polymorphism in a Turkish population (in healthy controls $2.8 \%$ and $0.5 \%$, respectively; in FMF patients $4.5 \%$ and $0.5 \%$, respectively) were also lower than those of Caucasian populations. In this study, PTPN22 gene polymorphism was not found to be a factor that could play a role in the pathogenesis and clinical course of FMF. Although there was no association between FMF and PTPN22 gene polymorphism in our study, the Th1-mediated inflammation in FMF could be caused by other factors independent of PTPN22related pathways.

However, this study has some limitations. First, studies of polymorphism require larger number of patients and controls compared to that of our study. Second, we used blood bank donors as healthy controls and this may have been a factor posing a risk of selection bias.

In conclusion, the distribution of PTPN22 C1858T polymorphism did not reveal any association with FMF in a Turkish population. Further studies are needed to evaluate the relationship between PTPN22 C1858T gene polymorphism and FMF.

\section{Declaration of conflicting interests}

The authors declared no conflicts of interest with respect to the authorship and/or publication of this article.

\section{Funding}

The study is supported by the Ankara University Research Fund.

\section{REFERENCES}

1. Ben-Chetrit E, Levy M. Familial Mediterranean fever. Lancet 1998;351:659-64.

2. Orbach $\mathrm{H}, \mathrm{Ben}-\mathrm{Ch}$ trit $\mathrm{E}$. Familial mediterranean fever - a review and update. Minerva Med 2001;92:421-30.

3. Centola M, Wood G, Frucht DM, Galon J, Aringer $\mathrm{M}$, Farrell $\mathrm{C}$, et al. The gene for familial Mediterranean fever, MEFV, is expressed in early leukocyte development and is regulated in response to inflammatory mediators. Blood 2000;95:3223-31.

4. Aypar E, Ozen S, Okur H, Kutluk T, Besbas N, Bakkaloglu A. Th1 polarization in familial Mediterranean fever. J Rheumatol 2003;30:2011-3.

5. Musabak U, Sengul A, Oktenli C, Pay S, Yesilova $\mathrm{Z}$, Kenar L, et al. Does immune activation continue during an attack-free period in familial Mediterranean fever? Clin Exp Immunol 2004;138:526-33.

6. Simsek I, Pay S, Pekel A, Dinc A, Musabak U, Erdem $\mathrm{H}$, et al. Serum proinflammatory cytokines directing $\mathrm{T}$ helper 1 polarization in patients with familial Mediterranean fever. Rheumatol Int 2007;27:807-11.

7. Criswell LA, Pfeiffer KA, Lum RF, Gonzales B, Novitzke J, Kern M, et al. Analysis of families in the multiple autoimmune disease genetics consortium (MADGC) collection: the PTPN22 620W allele associates with multiple autoimmune phenotypes. Am J Hum Genet 2005;76:561-71.

8. Bottini N, Musumeci L, Alonso A, Rahmouni S, Nika $\mathrm{K}$, Rostamkhani $\mathrm{M}$, et al. A functional variant of lymphoid tyrosine phosphatase is associated with type I diabetes. Nat Genet 2004;36:337-8.

9. Begovich $\mathrm{AB}$, Carlton VE, Honigberg LA, Schrodi SJ, Chokkalingam AP, Alexander $\mathrm{HC}$, et al. 
A missense single-nucleotide polymorphism in a gene encoding a protein tyrosine phosphatase (PTPN22) is associated with rheumatoid arthritis. Am J Hum Genet 2004;75:330-7.

10. Kyogoku C, Langefeld CD, Ortmann WA, Lee A, Selby S, Carlton VE, et al. Genetic association of the R620W polymorphism of protein tyrosine phosphatase PTPN22 with human SLE. Am J Hum Genet 2004;75:504-7.

11. Velaga MR, Wilson V, Jennings CE, Owen CJ, Herington S, Donaldson PT, et al. The codon 620 tryptophan allele of the lymphoid tyrosine phosphatase (LYP) gene is a major determinant of Graves' disease. $\mathrm{J}$ Clin Endocrinol Metab 2004;89:5862-5.

12. Livneh A, Langevitz P, Zemer D, Zaks N, Kees $\mathrm{S}$, Lidar $\mathrm{T}$, et al. Criteria for the diagnosis of familial Mediterranean fever. Arthritis Rheum 1997;40:1879-85.

13. Pras E, Livneh A, Balow JE Jr, Pras E, Kastner DL, Pras M, et al. Clinical differences between North African and Iraqi Jews with familial Mediterranean fever. Am J Med Genet 1998;75:216-9.

14. Begovich AB, Caillier SJ, Alexander HC, Penko JM, Hauser SL, Barcellos LF, et al. The R620W polymorphism of the protein tyrosine phosphatase PTPN22 is not associated with multiple sclerosis. Am J Hum Genet 2005;76:184-7.

15. Orozco G, García-Porrúa C, López-Nevot MA, Raya E, González-Gay MA, Martín J. Lack of association between ankylosing spondylitis and a functional polymorphism of PTPN22 proposed as a general susceptibility marker for autoimmunity. Ann Rheum Dis 2006;65:687-8.

16. Sahin N, Bicakcigil M, Atagunduz P, Direskeneli H, Saruhan-Direskeneli G. PTPN22 gene polymorphism in Behçet's disease. Tissue Antigens 2007;70:432-4.

17. Bakkaloglu A. Familial Mediterranean fever. Pediatr Nephrol 2003;18:853-9.

18. Vang $\mathrm{T}$, Miletic AV, Bottini N, Mustelin $\mathrm{T}$. Protein tyrosine phosphatase PTPN22 in human autoimmunity. Autoimmunity 2007;40:453-61.

19. Harrison P, Pointon JJ, Farrar C, Brown MA, Wordsworth BP. Effects of PTPN22 C1858T polymorphism on susceptibility and clinical characteristics of British Caucasian rheumatoid arthritis patients. Rheumatology (Oxford) 2006;45:1009-11.

20. Mori M, Yamada R, Kobayashi K, Kawaida R, Yamamoto K. Ethnic differences in allele frequency of autoimmune-disease-associated SNPs. J Hum Genet 2005;50:264-6.

21. Ikari K, Momohara S, Inoue E, Tomatsu T, Hara M, Yamanaka $\mathrm{H}$, et al. Haplotype analysis revealed no association between the PTPN22 gene and RA in a Japanese population. Rheumatology (Oxford) 2006;45:1345-8.

22. Ates A, Karaaslan Y, Karatayli E, Ertuğrul E, Aksaray $\mathrm{S}$, Türkyilmaz A, et al. Association of the PTPN22 gene polymorphism with autoantibody positivity in Turkish rheumatoid arthritis patients. Tissue Antigens 2011;78:56-9. 